\title{
A Generalization of Poly-Cauchy Numbers and Their Properties
}

\author{
Takao Komatsu, ${ }^{1}$ Vichian Laohakosol, ${ }^{2}$ and Kálmán Liptai ${ }^{3}$ \\ ${ }^{1}$ Graduate School of Science and Technology, Hirosaki University, Hirosaki 036-8561, Japan \\ ${ }^{2}$ Department of Mathematics, Faculty of Science, Kasetsart University, Bangkok 10900, Thailand \\ ${ }^{3}$ Institute of Mathematics and Informatics, Eszterházy Károly College, Eger 3300, Hungary
}

Correspondence should be addressed to Takao Komatsu; komatsu@cc.hirosaki-u.ac.jp

Received 23 April 2013; Revised 26 October 2013; Accepted 28 October 2013

Academic Editor: Gerd Teschke

Copyright (C) 2013 Takao Komatsu et al. This is an open access article distributed under the Creative Commons Attribution License, which permits unrestricted use, distribution, and reproduction in any medium, provided the original work is properly cited.

In Komatsu's work (2013), the concept of poly-Cauchy numbers is introduced as an analogue of that of poly-Bernoulli numbers. Both numbers are extensions of classical Cauchy numbers and Bernoulli numbers, respectively. There are several generalizations of poly-Cauchy numbers, including poly-Cauchy numbers with a $q$ parameter and shifted poly-Cauchy numbers. In this paper, we give a further generalization of poly-Cauchy numbers and investigate several arithmetical properties. We also give the corresponding generalized poly-Bernoulli numbers so that both numbers have some relations.

\section{Introduction}

Let $n \geq 0, k \geq 1$ be integers. Poly-Cauchy numbers of the first kind $c_{n}^{(k)}$ are defined by

$$
\begin{array}{r}
c_{n}^{(k)}=\underbrace{\int_{0}^{1} \cdots \int_{0}^{1}\left(x_{1} x_{2} \cdots x_{k}\right)\left(x_{1} x_{2} \cdots x_{k}-1\right) \cdots}_{k} \\
\left(x_{1} x_{2} \cdots x_{k}-n+1\right) d x_{1} d x_{2} \cdots d x_{k}
\end{array}
$$

[1]. The concept of poly-Cauchy numbers is a generalization of that of the classical Cauchy numbers $c_{n}=c_{n}^{(1)}$ defined by

$$
c_{n}=\int_{0}^{1} x(x-1) \cdots(x-n+1) d x
$$

(see, e.g., $[2,3])$. The generating function of poly-Cauchy numbers ([1], Theorem 2 ) is given by

$$
\operatorname{Lif}_{k}(\ln (1+x))=\sum_{n=0}^{\infty} c_{n}^{(k)} \frac{x^{n}}{n !},
$$

where

$$
\operatorname{Lif}_{k}(z)=\sum_{m=0}^{\infty} \frac{z^{m}}{m !(m+1)^{k}}
$$

is the kth polylogarithm factorial function. An explicit formula for $c_{n}^{(k)}([1]$, Theorem 1$)$ is given by

$$
c_{n}^{(k)}=\sum_{m=0}^{n}\left[\begin{array}{c}
n \\
m
\end{array}\right] \frac{(-1)^{n-m}}{(m+1)^{k}} \quad(n \geq 0, k \geq 1),
$$

where $\left[\begin{array}{c}n \\ m\end{array}\right]$ are the (unsigned) Stirling numbers of the first kind, arising as coefficients of the rising factorial

$$
x(x+1) \cdots(x+n-1)=\sum_{m=0}^{n}\left[\begin{array}{c}
n \\
m
\end{array}\right] x^{m}
$$

(see, e.g., [4]). See ([5], A224094-A224101) for the sequences arising from poly-Cauchy numbers.

The concept of poly-Cauchy numbers is an analogue of that of poly-Bernoulli numbers $B_{n}^{(k)}[6]$ defined by

$$
\frac{\mathrm{Li}_{k}\left(1-e^{-x}\right)}{1-e^{-x}}=\sum_{n=0}^{\infty} B_{n}^{(k)} \frac{x^{n}}{n !},
$$

where

$$
\operatorname{Li}_{k}(z)=\sum_{m=1}^{\infty} \frac{z^{m}}{m^{k}}
$$


is the $k$ th polylogarithm function. When $k=1, B_{n}=B_{n}^{(1)}$ is the classical Bernoulli number with $B_{1}^{(1)}=1 / 2$, defined by the generating function

$$
\frac{x e^{x}}{e^{x}-1}=\sum_{n=0}^{\infty} B_{n} \frac{x^{n}}{n !} .
$$

An explicit formula for $B_{n}^{(k)}([6]$, Theorem 1$)$ is given by

$$
B_{n}^{(k)}=(-1)^{n} \sum_{m=0}^{n}\left\{\begin{array}{l}
n \\
m
\end{array}\right\} \frac{(-1)^{m} m !}{(m+1)^{k}} \quad(n \geq 0, k \geq 1)
$$

where $\left\{\begin{array}{c}n \\ m\end{array}\right\}$ are the Stirling numbers of the second kind, determined by

$$
\left\{\begin{array}{c}
n \\
m
\end{array}\right\}=\frac{1}{m !} \sum_{j=0}^{m}(-1)^{j}\left(\begin{array}{c}
m \\
j
\end{array}\right)(m-j)^{n}
$$

(see, e.g., [4]).

There are some kinds of generalizations of poly-Cauchy numbers. One is the poly-Cauchy number with a q parame$\operatorname{ter} c_{n, q}^{(k)}$ [7] defined by

$$
\begin{aligned}
c_{n, q}^{(k)}=\underbrace{\int_{0}^{1} \cdots \int_{0}^{1}}_{k}\left(x_{1} \cdots x_{k}\right)\left(x_{1} \cdots x_{k}-q\right) \cdots \\
\quad\left(x_{1} \cdots x_{k}-(n-1) q\right) d x_{1} \cdots d x_{k} .
\end{aligned}
$$

Another is the shifted poly-Cauchy number $c_{n, a}^{(k)}$ [8] defined by

$$
\begin{aligned}
c_{n, a}^{(k)}=\underbrace{\int_{0}^{1} \cdots \int_{0}^{1}\left(x_{1} \cdots x_{k}\right)^{a}\left(x_{1} \cdots x_{k}-1\right) \cdots}_{k} \\
\quad\left(x_{1} \cdots x_{k}-(n-1)\right) d x_{1} \cdots d x_{k} .
\end{aligned}
$$

Notice that $c_{n, a}^{(k)}$ can be expressed as

$$
c_{n, a}^{(k)}=\sum_{m=0}^{n}\left[\begin{array}{c}
n \\
m
\end{array}\right] \frac{(-1)^{n-m}}{(m+a)^{k}} .
$$

For example, if $n=5$ and $a=3$, then

$$
\begin{aligned}
& c_{5}^{(k)}=\frac{24}{2^{k}}-\frac{50}{3^{k}}+\frac{35}{4^{k}}-\frac{10}{5^{k}}+\frac{1}{6^{k}}, \\
& c_{5,3}^{(k)}=\frac{24}{4^{k}}-\frac{50}{5^{k}}+\frac{35}{6^{k}}-\frac{10}{7^{k}}+\frac{1}{8^{k}} .
\end{aligned}
$$

Therefore, such numbers are shifted from the original polyCauchy numbers. Remember that the Hurwitz zeta function $\zeta(s, q)=\sum_{n=0}^{\infty} 1 /(q+n)^{s}$ is a generalization of the famous Riemann zeta function $\zeta(s)=\sum_{n=1}^{\infty} 1 / n^{s}$ since $\zeta(s)=\zeta(s, 1)$.

In this paper, we give a further generalization of polyCauchy numbers, including both kinds of generalizations, and show several combinatorial and characteristic properties. We also give the corresponding poly-Bernoulli numbers so that both numbers have some relations.

\section{Definitions and Basic Properties}

Let $n \geq 0, k \geq 1$ be integers, and let $a, q$ and $l_{1}, \ldots, l_{k}$ be nonzero real numbers. For simplicity, we write $L=\left(l_{1}, \ldots, l_{k}\right)$ and $\ell=l_{1} \cdots l_{k}$. Define $c_{n, a, q, L}^{(k)}$ by

$$
\begin{aligned}
c_{n, a, q, L}^{(k)}=\underbrace{\int_{0}^{l_{1}} \cdots \int_{0}^{l_{k}}\left(x_{1} \cdots x_{k}\right)^{a}\left(x_{1} \cdots x_{k}-q\right) \cdots}_{k} \\
\left(x_{1} \cdots x_{k}-(n-1) q\right) d x_{1} \cdots d x_{k} .
\end{aligned}
$$

Then, $c_{n, a, q, L}^{(k)}$ can be expressed in terms of the Stirling numbers of the first kind $\left[\begin{array}{c}n \\ m\end{array}\right]$.

Theorem 1. Let a be a positive real number. Then,

$$
c_{n, a, q, L}^{(k)}=\sum_{m=0}^{n}\left[\begin{array}{c}
n \\
m
\end{array}\right] \frac{(-q)^{n-m} e^{m+a}}{(m+a)^{k}} \quad(n \geq 0, k \geq 1) .
$$

Remark 2. If $a=\ell=1$, then $c_{n, 1, q,(1, \ldots, 1)}^{(k)}=c_{n, q}^{(k)}$ is the poly-Cauchy number with a $q$ parameter ([7], Theorem 1$)$. If $q=\ell=1$, then $c_{n, a, 1,(1, \ldots, 1)}^{(k)}=c_{n, a}^{(k)}$ is the shifted poly-Cauchy number ([8], Theorem 2$)$.

Proof. By

$$
x(x-1) \cdots(x-n+1)=\sum_{m=0}^{n}\left[\begin{array}{c}
n \\
m
\end{array}\right](-1)^{n-m} x^{m},
$$

we have

$$
\begin{aligned}
c_{n, a, q, L}^{(k)}= & \int_{0}^{l_{1}} \ldots \int_{0}^{l_{k}} \sum_{m=0}^{n}\left[\begin{array}{c}
n \\
m
\end{array}\right](-1)^{n-m} \\
& \quad \times\left(x_{1} \cdots x_{k}\right)^{m+a-1} q^{n-m} d x_{1} \cdots d x_{k} \\
= & \sum_{m=0}^{n}\left[\begin{array}{c}
n \\
m
\end{array}\right] \frac{(-q)^{n-m} \ell^{m+a}}{(m+a)^{k}} .
\end{aligned}
$$

For an integer $k$ and a positive real number $a$, define the extended polylogarithm factorial function $\operatorname{Lif}_{k}(z ; a)$ by

$$
\operatorname{Lif}_{k}(z ; a)=\sum_{m=0}^{\infty} \frac{z^{m}}{m !(m+a)^{k}}
$$

[8]. When $a=1, \operatorname{Lif}_{k}(z ; 1)=\operatorname{Lif}_{k}(z)$ is the polylogarithm factorial function [1]. The generating function of the number $c_{n, a, q, L}^{(k)}(q \neq 0)$ is given by using the extended polylogarithm factorial function $\operatorname{Lif}_{k}(a ; z)$.

Theorem 3. One has

$$
\ell^{a} \operatorname{Lif}_{k}\left(\frac{\ell \ln (1+q x)}{q} ; a\right)=\sum_{n=0}^{\infty} c_{n, a, q, L}^{(k)} \frac{x^{n}}{n !} .
$$


Remark 4. If $a=\ell=1$, then Theorem 3 is reduced to Theorem 2 in [7]. If $q=\ell=1$, then Theorem 3 is reduced to Theorem 3 in [8].

Proof. Since

$$
\frac{(\ln (1+x))^{m}}{m !}=(-1)^{m} \sum_{n=m}^{\infty}\left[\begin{array}{c}
n \\
m
\end{array}\right] \frac{(-x)^{n}}{n !}
$$

by Theorem 1 we have

$$
\begin{aligned}
\sum_{n=0}^{\infty} c_{n, a, q, L}^{(k)} \frac{x^{n}}{n !} & =\sum_{n=0}^{\infty} \sum_{m=0}^{n}\left[\begin{array}{c}
n \\
m
\end{array}\right] \frac{(-q)^{n-m} \ell^{m+a}}{(m+a)^{k}} \frac{x^{n}}{n !} \\
& =\ell^{a} \sum_{m=0}^{\infty} \frac{(-q)^{-m} \ell^{m}}{(m+a)^{k}} \sum_{n=m}^{\infty}\left[\begin{array}{c}
n \\
m
\end{array}\right] \frac{(-q x)^{n}}{n !} \\
& =\ell^{a} \sum_{m=0}^{\infty} \frac{(-q)^{-m} \ell^{m}}{(m+a)^{k}}(-1)^{m} \frac{(\ln (1+q x))^{m}}{m !} \\
& =\ell^{a} \sum_{m=0}^{\infty} \frac{1}{m !(m+a)^{k}}\left(\frac{\ell \ln (1+q x)}{q}\right)^{m} \\
& =\ell^{a} \operatorname{Lif}_{k}\left(\frac{\ell \ln (1+q x)}{q} ; a\right) .
\end{aligned}
$$

The generating function of the number $c_{n, a, q, L}^{(k)}$ can be written in the form of iterated integrals.

Corollary 5. Let $a$ and $q$ be real numbers with $a>0$ and $q \neq 0$. For $k=1$, one has

$$
\begin{aligned}
& \left(\frac{\ell q}{\ln (1+q x)}\right)^{a} \int_{0}^{x}\left(\frac{\ln (1+q x)}{q}\right)^{a-1}(1+q x)^{\ell / q-1} d x \\
& =\sum_{n=0}^{\infty} c_{n, a, q, L}^{(1)} \frac{x^{n}}{n !}
\end{aligned}
$$

For $k>1$, one has

$$
\begin{aligned}
& \left(\frac{\ell q}{\ln (1+q x)}\right) \\
& \underbrace{\int_{0}^{x} \frac{q}{(1+q x) \ln (1+q x)} \int_{0}^{x} \cdots \frac{q}{(1+q x) \ln (1+q x)} \int_{0}^{x}}_{k} \\
& \left(\frac{\ln (1+q x)}{q}\right)^{a-1}(1+q x)^{\ell / q-1} \underbrace{\frac{d x \cdots d x}{\left(1+\sum_{n=0} c_{n, a, q, L}^{(k)}\right.} \frac{x^{n}}{n !}}_{k} .
\end{aligned}
$$

Remark 6. If $a=\ell=1$, then Corollary 5 is reduced to Corollary 1 in [7]. If $q=\ell=1$, then Corollary 5 is reduced to Corollary 1 in [8].
Proof. For $k=1$,

$$
\begin{aligned}
\operatorname{Lif}_{1}(z ; a)= & \sum_{m=0}^{\infty} \frac{z^{m}}{m !(m+a)}=\frac{1}{z^{a}} \sum_{m=0}^{\infty} \frac{z^{m+a}}{m !(m+a)} \\
= & \frac{1}{z^{a}} \int_{0}^{z} \sum_{m=0}^{\infty} \frac{z^{m+a-1}}{m !}=\frac{1}{z^{a}} \int_{0}^{z} z^{a-1} e^{z} d z \\
= & \frac{1}{z^{a}}\left((-1)^{a}(a-1) !\right. \\
& \left.+e^{z} \sum_{i=0}^{a-1}(-1)^{i} \frac{(a-1) !}{(a-i-1) !} z^{a-i-1}\right) .
\end{aligned}
$$

Note that the last equation holds only if $a$ is an integer. For $k>1$, we have

$$
\begin{aligned}
\operatorname{Lif}_{k}(z ; a) & =\frac{1}{z^{a}} \sum_{m=0}^{\infty} \frac{z^{m+a}}{m !(m+a)^{k}} \\
& =\frac{1}{z^{a}} \int_{0}^{z} \sum_{m=0}^{\infty} \frac{z^{m+a-1}}{m !(m+a)^{k-1}} d z \\
& =\frac{1}{z^{a}} \int_{0}^{z} z^{a-1} \operatorname{Lif}_{k-1}(z ; a) d z .
\end{aligned}
$$

Hence,

$$
\operatorname{Lif}_{k}(z ; a)=\frac{1}{z^{a}} \underbrace{\int_{0}^{z} \frac{1}{z} \int_{0}^{z} \cdots \frac{1}{z} \int_{0}^{z} \frac{1}{z} \int_{0}^{z} z^{a-1}}_{k} e^{z} \underbrace{d z \cdots d z}_{k} .
$$

Putting $z=\ell \ln (1+q x) / q$ and multiplying by $\ell^{a}$, we get the result.

\section{Poly-Cauchy Numbers of the Second Kind}

In [1], the concept of poly-Cauchy numbers of the second kind is also introduced. The poly-Cauchy numbers of the second kind $\hat{c}_{n}^{(k)}$ are defined by

$$
\begin{array}{r}
\hat{c}_{n}^{(k)}=\underbrace{\int_{0}^{1} \cdots \int_{0}^{1}}_{k}\left(-x_{1} x_{2} \cdots x_{k}\right)\left(-x_{1} x_{2} \cdots x_{k}-1\right) \cdots \\
\left(-x_{1} x_{2} \cdots x_{k}-n+1\right) d x_{1} d x_{2} \cdots d x_{k},
\end{array}
$$

and the generating function is given by

$$
\operatorname{Lif}_{k}(-\ln (1+x))=\sum_{n=0}^{\infty} \widehat{c}_{n}^{(k)} \frac{x^{n}}{n !}
$$

Then, the poly-Cauchy numbers of the second kind $\widehat{c}_{n}^{(k)}$ can also be expressed in terms of the Stirling numbers of the first kind ([1], Theorem 4). See ([5], A219247, A224102A224107, A224109) for the sequences arising from polyCauchy numbers of the second kind.

Proposition 7. One has

$$
\widehat{c}_{n}^{(k)}=(-1)^{n} \sum_{m=0}^{n}\left[\begin{array}{l}
n \\
m
\end{array}\right] \frac{1}{(m+1)^{k}} .
$$


Let $a$ be a positive real number. Similar to generalized poly-Cauchy numbers of the first kind $c_{n, a, q, L}^{(k)}$, define the polyCauchy numbers of the second kind $\widehat{c}_{n, a, q, L}^{(k)}(n \geq 0, k \geq 1)$ by

$$
\begin{array}{r}
\widehat{c}_{n, a, q, L}^{(k)}=(-1)^{a-1} \int_{0}^{l_{1}} \cdots \int_{0}^{l_{k}}\left(-x_{1} \cdots x_{k}\right)^{a}\left(-x_{1} \cdots x_{k}-q\right) \cdots \\
\left(-x_{1} \cdots x_{k}-(n-1) q\right) d x_{1} \cdots d x_{k} .
\end{array}
$$

Then, similar to Theorem $1, \widehat{c}_{n, a, q, L}^{(k)}$ can also be expressed in terms of the Stirling numbers of the first kind $\left[\begin{array}{c}n \\ m\end{array}\right]$.

Theorem 8. One has

$$
\widehat{c}_{n, a, q, L}^{(k)}=(-1)^{n} \sum_{m=0}^{n}\left[\begin{array}{c}
n \\
m
\end{array}\right] \frac{q^{n-m} \ell^{m+a}}{(m+a)^{k}} \quad(n \geq 0, k \geq 1) .
$$

Theorem 9. The generating function of the number $\widehat{c}_{n, a, q, L}^{(k)}$ is given by

$$
\ell^{a} \operatorname{Lif}_{k}\left(-\frac{\ell \ln (1+q x)}{q} ; a\right)=\sum_{m=0}^{\infty} \widehat{c}_{n, a, q, L}^{(k)} \frac{x^{n}}{n !},
$$

where

$$
\operatorname{Lif}_{k}(z ; a)=\sum_{m=0}^{\infty} \frac{z^{m}}{m !(m+a)^{k}} .
$$

Remark 10. If $a=\ell=1$, then Theorem 8 is reduced to Theorem 3 in [7] and Theorem 9 is reduced to Theorem 4 in [7]. If $q=\ell=1$, then Theorem 8 is reduced to Theorem 5 in [8] and Theorem 9 is reduced to Theorem 6 in [8].

The generating function of the number $\widehat{c}_{n, a, q, L}^{(k)}$ can be written in the form of iterated integrals.

Corollary 11. Let a be a positive real number. For $k=1$, one has

$$
\begin{aligned}
& \left(\frac{\ell q}{\ln (1+q x)}\right)^{a} \int_{0}^{x}\left(\frac{\ln (1+q x)}{q}\right)^{a-1}(1+q x)^{-\ell / q-1} d x \\
& =\sum_{n=0}^{\infty} \widehat{c}_{n, a, q, L}^{(1)} \frac{x^{n}}{n !} .
\end{aligned}
$$

For $k>1$, one has

$$
\begin{aligned}
& \left(\frac{\ell q}{\ln (1+q x)}\right) \\
& \underbrace{\int_{0}^{x} \frac{q}{(1+q x) \ln (1+q x)} \int_{0}^{x} \cdots \frac{q}{(1+q x) \ln (1+q x)} \int_{0}^{x}}_{k} \\
& \left(\frac{\ln (1+q x)}{q}\right)^{a-1}(1+q x)^{-\ell / q-1} \frac{d x \cdots d x}{k}=\sum_{n=0}^{\infty} \widehat{c}_{n, a, q, L}^{(k)} \frac{x^{n}}{n !} .
\end{aligned}
$$

Remark 12. When $a=q=k=\ell=1$ in the first identity, we have the generating function of the classical Cauchy numbers of the second kind:

$$
\frac{x}{(1+x) \ln (1+x)}=\sum_{n=0}^{\infty} \widehat{c}_{n} \frac{x^{n}}{n !} .
$$

In addition, there are relations between both kinds of poly-Cauchy numbers if $q=1$. For simplicity, we write $c_{n, a, L}^{(k)}=$ $c_{n, a, 1, L}^{(k)}$ and $\widehat{c}_{n, a, L}^{(k)}=\widehat{c}_{n, a, 1, L}^{(k)}$.

Theorem 13. Let $k$ be an integer and a a positive real number. For $n \geq 1$, one has

$$
\begin{aligned}
& (-1)^{n} \frac{c_{n, a, L}^{(k)}}{n !}=\sum_{m=1}^{n}\left(\begin{array}{c}
n-1 \\
m-1
\end{array}\right) \frac{\widehat{c}_{m, a, L}^{(k)}}{m !}, \\
& (-1)^{n} \frac{\widehat{c}_{n, a, L}^{(k)}}{n !}=\sum_{m=1}^{n}\left(\begin{array}{c}
n-1 \\
m-1
\end{array}\right) \frac{c_{m, a, L}^{(k)}}{m !} .
\end{aligned}
$$

Remark 14. If $a=\ell=1$, then Theorem 13 is reduced to Theorem 7 in [1].

Proof. We will prove the second identity. The first one is proved similarly and omitted. By using the identity (see, e.g., [4], Chapter 6)

$$
\frac{(-1)^{i}}{n !}\left[\begin{array}{c}
n \\
i
\end{array}\right]=\sum_{m=1}^{n} \frac{(-1)^{m}}{m !}\left(\begin{array}{c}
n-1 \\
m-1
\end{array}\right)\left[\begin{array}{c}
m \\
i
\end{array}\right]
$$

and Theorems 1 and 8 , we have

$$
\begin{aligned}
\text { RHS } & =\sum_{m=1}^{n}\left(\begin{array}{c}
n-1 \\
m-1
\end{array}\right) \frac{1}{m !} \\
& \times \sum_{i=1}^{m}\left[\begin{array}{c}
m \\
i
\end{array}\right] \frac{(-1)^{m-i} e^{i+a}}{(i+a)^{k}} \\
= & \sum_{i=1}^{n} \frac{(-1)^{i} \ell^{i}}{(i+a)^{k}} \sum_{m=i}^{n} \frac{(-1)^{m}}{m !}\left(\begin{array}{c}
n-1 \\
m-1
\end{array}\right)\left[\begin{array}{c}
m \\
i
\end{array}\right] \\
= & \sum_{i=1}^{n} \frac{(-1)^{i} \ell^{i+a}}{(i+a)^{k}} \frac{(-1)^{i}}{n !}\left[\begin{array}{c}
n \\
i
\end{array}\right]=\text { LHS. }
\end{aligned}
$$

\section{Some Expressions of Poly-Cauchy Numbers with Negative Indices}

It is known that poly-Bernoulli numbers satisfy the duality theorem $B_{n}^{(-k)}=B_{k}^{(-n)}$ for $n, k \geq 0$ ([6], Theorem 2) because of the symmetric formula

$$
\sum_{n=0}^{\infty} \sum_{k=0}^{\infty} B_{n}^{(-k)} \frac{x^{n}}{n !} \frac{y^{k}}{k !}=\frac{e^{x+y}}{e^{x}+e^{y}-e^{x+y}} .
$$

However, the corresponding duality theorem does not hold for poly-Cauchy numbers for any real number $a$, by the following results. 
Proposition 15. Suppose that $\ell=1$. Then, for nonnegative integers $n$ and $k$ and $a$ real number $a \neq 0$, one has

$$
\begin{aligned}
& \sum_{n=0}^{\infty} \sum_{k=0}^{\infty} c_{n, a, q, L}^{(-k)} \frac{x^{n}}{n !} \frac{y^{k}}{k !}=e^{a y}(1+q x)^{e^{y} / q}, \\
& \sum_{n=0}^{\infty} \sum_{k=0}^{\infty} \widehat{c}_{n, a, q, L}^{(-k)} \frac{x^{n}}{n !} \frac{y^{k}}{k !}=\frac{e^{a y}}{(1+q x)^{e^{y} / q}}
\end{aligned}
$$

Remark 16. If $a=\ell=1$, then Proposition 15 is reduced to Proposition 1 in [7]. If $q=\ell=1$, then Proposition 15 is reduced to Proposition 3 in [8].

Proof. We will prove the first identity. The second identity is proved similarly. By Theorem 3, we have

$$
\begin{aligned}
\sum_{n=0}^{\infty} \sum_{k=0}^{\infty} c_{n, a, q, L}^{(-k)} \frac{x^{n}}{n !} \frac{y^{k}}{k !} & \\
& =\sum_{k=0}^{\infty}\left(\sum_{n=0}^{\infty} c_{n, a, q, L}^{(-k)} \frac{x^{n}}{n !}\right) \frac{y^{k}}{k !} \\
& =\sum_{k=0}^{\infty} \ell^{a} \sum_{m=0}^{\infty} \frac{(m+a)^{k}}{m !}\left(\frac{\ell \ln (1+q x)}{q}\right)^{m} \frac{y^{k}}{k !} \\
& =\sum_{m=0}^{\infty} \frac{1}{m !}\left(\frac{\ln (1+q x)}{q}\right)^{m} \sum_{k=0}^{\infty} \frac{((m+a) y)^{k}}{k !} \\
& =\sum_{m=0}^{\infty} \frac{1}{m !}\left(\frac{\ln (1+q x)}{q}\right)^{m} e^{(m+a) y} \\
& =e^{a y} \sum_{m=0}^{\infty} \frac{1}{m !}\left(\frac{e^{y}}{q} \ln (1+q x)\right)^{m}=e^{a y}(1+q x)^{e^{y} / q}
\end{aligned}
$$

By using Proposition 15, we have explicit expressions of poly-Cauchy numbers with negative indices. For simplicity, we write $c_{n, a, q}^{(-k)}=c_{n, a, q, L}^{(-k)}$ and $\widehat{c}_{n, a, q}^{(-k)}=\widehat{c}_{n, a, q, L}^{(-k)}$ if $\ell=1$.

Theorem 17. For nonnegative integers $n, k$, and a real number $a \neq 0$, one has

$$
\begin{array}{r}
c_{n, a, q}^{(-k)}=\sum_{i=0}^{k} \sum_{j=0}^{i} \sum_{\lambda=0}^{n} \sum_{\nu=0}^{\lambda} j !\left(\begin{array}{c}
k \\
i
\end{array}\right)\left\{\begin{array}{l}
i \\
j
\end{array}\right\}\left(\begin{array}{c}
n \\
\lambda
\end{array}\right)\left[\begin{array}{c}
n-\lambda \\
j
\end{array}\right] \\
\times\left[\begin{array}{c}
\lambda \\
v
\end{array}\right] a^{k-i}(-q)^{n-j-\nu}, \\
\widehat{c}_{n, a, q}^{(-k)}=\sum_{i=0}^{k} \sum_{j=0}^{i} \sum_{\lambda=0}^{n} \sum_{\nu=0}^{\lambda}(-1)^{n} j !\left(\begin{array}{c}
k \\
i
\end{array}\right)\left\{\begin{array}{l}
i \\
j
\end{array}\right\}\left(\begin{array}{c}
n \\
\lambda
\end{array}\right) \\
\times\left[\begin{array}{c}
n-\lambda \\
j
\end{array}\right]\left[\begin{array}{l}
\lambda \\
\nu
\end{array}\right] a^{k-i} q^{n-j-v} .
\end{array}
$$

Remark 18. If $a=q=1$, by

$$
\sum_{i=0}^{k}\left(\begin{array}{l}
k \\
i
\end{array}\right)\left\{\begin{array}{l}
i \\
j
\end{array}\right\}=\left\{\begin{array}{l}
k+1 \\
j+1
\end{array}\right\}
$$

[4], the above identities become

$$
\begin{aligned}
c_{n, 1,1}^{(-k)}= & c_{n}^{(-k)} \\
= & \sum_{j=0}^{k}(-1)^{n+j} j ! \\
& \times\left(\left[\begin{array}{c}
n \\
j
\end{array}\right]-n\left[\begin{array}{c}
n-1 \\
j
\end{array}\right]\right)\left\{\begin{array}{c}
k+1 \\
j+1
\end{array}\right\}, \\
\widehat{c}_{n, 1,1}^{(-k)}=\widehat{c}_{n}^{(-k)}= & \sum_{j=0}^{k}(-1)^{n} j !\left[\begin{array}{c}
n+1 \\
j+1
\end{array}\right]\left\{\begin{array}{c}
k+1 \\
j+1
\end{array}\right\} .
\end{aligned}
$$

Proof. By Proposition 15 together with

$$
\begin{gathered}
\frac{\left(e^{y}-1\right)^{j}}{j !}=\sum_{k=j}^{\infty}\left\{\begin{array}{c}
k \\
j
\end{array}\right\} \frac{y^{k}}{k !}, \\
\frac{(-\ln (1+x))^{j}}{j !}=\sum_{n=j}^{\infty}\left[\begin{array}{l}
n \\
j
\end{array}\right] \frac{(-x)^{n}}{n !}
\end{gathered}
$$

[4], we have

$$
\begin{aligned}
\sum_{n=0}^{\infty} \sum_{k=0}^{\infty} c_{n, a, q}^{(-k)} \frac{x^{n}}{n !} \frac{y^{k}}{k !} & \left((1+q x)^{1 / q}\right)^{e^{y}-1}(1+q x)^{1 / q} e^{a y} \\
= & \exp \left(\left(e^{y}-1\right)(\ln (1+q x))^{1 / q}\right) \\
& \times(1+q x)^{1 / q} e^{a y} \\
= & \sum_{j=0}^{\infty} \frac{j !}{q^{j}} \frac{\left(e^{y}-1\right)^{j}}{j !} \frac{(\ln (1+q x))^{j}}{j !} \\
& \times(1+q x)^{1 / q} e^{a y} \\
= & \sum_{j=0}^{\infty}(-1)^{j} \frac{j !}{q^{j}} e^{a y} \sum_{k=j}^{\infty}\left\{\begin{array}{c}
k \\
k
\end{array}\right\} \frac{y^{k}}{k !}(1+q x)^{1 / q} \\
& \times \sum_{n=j}^{\infty}\left[\begin{array}{c}
n \\
j
\end{array}\right] \frac{(-q x)^{n}}{n !} .
\end{aligned}
$$


Since

$e^{a y} \sum_{k=j}^{\infty}\left\{\begin{array}{c}k \\ j\end{array}\right\} \frac{y^{k}}{k !}=\sum_{l=0}^{\infty} \frac{(a y)^{l}}{l !} \sum_{k=j}^{\infty}\left\{\begin{array}{c}k \\ j\end{array}\right\} \frac{y^{k}}{k !}$

$$
\begin{aligned}
& =\sum_{k=0}^{\infty}\left(\sum_{i=0}^{k} \frac{a^{k-i}}{(k-i) !}\left\{\begin{array}{l}
i \\
j
\end{array}\right\} \frac{1}{i !}\right) y^{k} \\
& =\sum_{k=0}^{\infty}\left(\sum_{i=0}^{k}\left(\begin{array}{l}
k \\
i
\end{array}\right)\left\{\begin{array}{l}
i \\
j
\end{array}\right\} a^{k-i}\right) \frac{y^{k}}{k !}, \\
& (1+q x)^{1 / q} \sum_{n=j}^{\infty}\left[\begin{array}{l}
n \\
j
\end{array}\right] \frac{(-q x)^{n}}{n !} \\
& =\sum_{l=0}^{\infty}\left(\begin{array}{c}
\frac{1}{q} \\
l
\end{array}\right)(q x)^{l} \sum_{n=j}^{\infty}\left[\begin{array}{l}
n \\
j
\end{array}\right] \frac{(-q x)^{n}}{n !} \\
& =\sum_{l=0}^{\infty}(-1)^{l} \sum_{\nu=0}^{l}\left[\begin{array}{l}
l \\
\nu
\end{array}\right]\left(-\frac{1}{q}\right)^{\nu} \frac{(q x)^{l}}{l !} \\
& \times \sum_{n=j}^{\infty}\left[\begin{array}{c}
n \\
j
\end{array}\right] \frac{(-q x)^{n}}{n !} \\
& =\sum_{l=0}^{\infty} \sum_{\nu=0}^{l}\left[\begin{array}{l}
l \\
v
\end{array}\right](-q)^{l-\nu} \frac{x^{l}}{l !} \sum_{n=0}^{\infty}\left[\begin{array}{l}
n \\
j
\end{array}\right] \frac{(-q x)^{n}}{n !} \\
& =\sum_{n=0}^{\infty} \sum_{\lambda=0}^{n} \sum_{\nu=0}^{\lambda}\left[\begin{array}{l}
\lambda \\
v
\end{array}\right] \frac{(-q)^{\lambda-\nu}}{\lambda !}\left[\begin{array}{c}
n-\lambda \\
j
\end{array}\right] \frac{(-q)^{n-\lambda}}{(n-\lambda) !} x^{n} \\
& =\sum_{n=0}^{\infty} \sum_{\lambda=0}^{n}\left(\begin{array}{l}
n \\
\lambda
\end{array}\right)\left[\begin{array}{c}
n-\lambda \\
j
\end{array}\right] \sum_{\nu=0}^{\lambda}\left[\begin{array}{l}
\lambda \\
\nu
\end{array}\right](-q)^{n-\nu} \frac{x^{n}}{n !},
\end{aligned}
$$

we obtain

$$
\begin{aligned}
& \sum_{n=0}^{\infty} \sum_{k=0}^{\infty} c_{n, a, q}^{(-k)} \frac{x^{n}}{n !} \frac{y^{k}}{k !} \sum_{n=0}^{\infty} \sum_{k=0}^{\infty} \sum_{i=0}^{k} \sum_{j=0}^{i} \frac{(-1)^{j} j !}{q^{j}}\left(\begin{array}{c}
k \\
i
\end{array}\right)\left\{\begin{array}{l}
i \\
j
\end{array}\right\} a^{k-i} \\
& \times \sum_{\lambda=0}^{n}\left(\begin{array}{c}
n \\
\lambda
\end{array}\right)\left[\begin{array}{c}
n-\lambda \\
j
\end{array}\right] \sum_{\nu=0}^{\lambda}\left[\begin{array}{l}
\lambda \\
v
\end{array}\right](-q)^{n-\nu} \frac{x^{n}}{n !} \frac{y^{k}}{k !} \\
&= \sum_{n=0}^{\infty} \sum_{k=0}^{\infty} \sum_{i=0}^{k} \sum_{j=0}^{i} \sum_{\lambda=0}^{n} \sum_{\nu=0}^{\lambda} j !\left(\begin{array}{c}
k \\
i
\end{array}\right)\left\{\begin{array}{l}
i \\
j
\end{array}\right\} \\
& \times\left(\begin{array}{c}
n \\
\lambda
\end{array}\right)\left[\begin{array}{c}
n-\lambda \\
j
\end{array}\right]\left[\begin{array}{l}
\lambda \\
\nu
\end{array}\right] a^{k-i} \\
& \times(-q)^{n-j-\nu} \frac{x^{n}}{n !} \frac{y^{k}}{k !} .
\end{aligned}
$$

$$
\sum_{n=0}^{\infty} \sum_{k=0}^{\infty} \widehat{c}_{n, a, q}^{(-k)} \frac{x^{n}}{n !} \frac{y^{k}}{k !}
$$

$$
\begin{aligned}
& (1+q x)^{-1 / q} \sum_{n=j}^{\infty}\left[\begin{array}{c}
n \\
j
\end{array}\right] \frac{(-q x)^{n}}{n !} \\
& =\sum_{l=0}^{\infty}(-1)^{l} \sum_{\nu=0}^{l}\left[\begin{array}{l}
l \\
v
\end{array}\right]\left(\frac{1}{q}\right)^{\nu} \frac{(q x)^{l}}{l !} \\
& \times \sum_{n=j}^{\infty}\left[\begin{array}{l}
n \\
j
\end{array}\right] \frac{(-q x)^{n}}{n !} \\
& =\sum_{l=0}^{\infty} \sum_{\nu=0}^{l}\left[\begin{array}{l}
l \\
v
\end{array}\right] q^{l-v}(-1)^{l} \frac{x^{l}}{l !} \sum_{n=0}^{\infty}\left[\begin{array}{l}
n \\
j
\end{array}\right] \frac{(-q x)^{n}}{n !} \\
& =\sum_{n=0}^{\infty} \sum_{\lambda=0}^{n} \sum_{v=0}^{\lambda}\left[\begin{array}{l}
\lambda \\
v
\end{array}\right] \frac{q^{\lambda-\nu}(-1)^{\lambda}}{\lambda !} \\
& \times\left[\begin{array}{c}
n-\lambda \\
j
\end{array}\right] \frac{(-q)^{n-\lambda}}{(n-\lambda) !} x^{n} \\
& =\sum_{n=0}^{\infty}(-1)^{n} \sum_{\lambda=0}^{n}\left(\begin{array}{l}
n \\
\lambda
\end{array}\right)\left[\begin{array}{c}
n-\lambda \\
j
\end{array}\right] \sum_{\nu=0}^{\lambda}\left[\begin{array}{l}
\lambda \\
\nu
\end{array}\right] q^{n-\nu} \frac{x^{n}}{n !},
\end{aligned}
$$

we get

$$
\begin{aligned}
= & (1+q x)^{-e^{y} / q} e^{a y} \\
= & \exp \left(\left(e^{y}-1\right)(\ln (1+q x))^{-1 / q}\right) \\
& \times(1+q x)^{-1 / q} e^{a y} \\
= & \sum_{j=0}^{\infty} \frac{j !}{q^{j}} \frac{\left(e^{y}-1\right)^{j}}{j !} \frac{(-\ln (1+q x))^{j}}{j !} \\
& \times(1+q x)^{-1 / q} e^{a y} \\
= & \sum_{j=0}^{\infty} \frac{j !}{q^{j}} e^{a y} \sum_{k=j}^{\infty}\left\{\begin{array}{l}
k \\
k
\end{array}\right\} \frac{y^{k}}{k !}(1+q x)^{-1 / q} \\
& \times \sum_{n=j}^{\infty}\left[\begin{array}{c}
n \\
j
\end{array}\right] \frac{(-q x)^{n}}{n !} \\
= & \sum_{j=0}^{\infty} \frac{j !}{q^{j}} \sum_{k=0}^{\infty}\left(\begin{array}{c}
k \\
\left.\sum_{i=0}\left(\begin{array}{l}
k \\
i
\end{array}\right)\left\{\begin{array}{l}
i \\
j
\end{array}\right\} a^{k-i}\right) \frac{y^{k}}{k !}
\end{array}\right.
\end{aligned}
$$




$$
\begin{aligned}
\times \sum_{n=0}^{\infty}(-1)^{n} \sum_{\lambda=0}^{n}\left(\begin{array}{l}
n \\
\lambda
\end{array}\right)\left[\begin{array}{c}
n-\lambda \\
j
\end{array}\right] \sum_{\nu=0}^{\lambda}\left[\begin{array}{l}
\lambda \\
v
\end{array}\right] q^{n-\nu} \frac{x^{n}}{n !} \\
=\sum_{n=0}^{\infty} \sum_{k=0}^{\infty} \sum_{i=0}^{k} \sum_{j=0}^{i} \sum_{\lambda=0}^{n} \sum_{v=0}^{\lambda}(-1)^{n} j ! \\
\times\left(\begin{array}{c}
k \\
i
\end{array}\right)\left\{\begin{array}{l}
i \\
j
\end{array}\right\}\left(\begin{array}{c}
n \\
\lambda
\end{array}\right)\left[\begin{array}{c}
n-\lambda \\
j
\end{array}\right] \\
\times\left[\begin{array}{c}
\lambda \\
v
\end{array}\right] a^{k-i} q^{n-j-v} \frac{x^{n}}{n !} \frac{y^{k}}{k !} .
\end{aligned}
$$

\section{Poly-Bernoulli Numbers Corresponding to Poly-Cauchy Numbers}

In this section, we will consider the corresponding generalized poly-Bernoulli numbers to the generalized poly-Cauchy numbers discussed in the previous sections. Let $k$ be an integer and $a$ a positive real number. An explicit form of polyBernoulli number $B_{n}^{(k)}$ is given by

$$
B_{n}^{(k)}=\sum_{m=0}^{n}\left\{\begin{array}{l}
n \\
m
\end{array}\right\} \frac{(-1)^{n-m} m !}{(m+1)^{k}}
$$

([6], Theorem 1). In ([1], Theorem 8), one expression of $B_{n}^{(k)}$ in terms of poly-Cauchy numbers $c_{n}^{(k)}$ is given.

Proposition 19. One has

$$
B_{n}^{(k)}=\sum_{l=1}^{n} \sum_{m=1}^{n} m !\left\{\begin{array}{c}
n \\
m
\end{array}\right\}\left\{\begin{array}{c}
m-1 \\
l-1
\end{array}\right\} c_{l}^{(k)} \quad(n \geq 1) .
$$

On the contrary, in ([9], Theorem 2.2), one expression of $c_{n}^{(k)}$ in terms of $B_{n}^{(k)}$ is given.

Proposition 20. One has

$$
c_{n}^{(k)}=\sum_{l=1}^{n} \sum_{m=1}^{n} \frac{(-1)^{n-m}}{m !}\left[\begin{array}{c}
n \\
m
\end{array}\right]\left[\begin{array}{c}
m \\
l
\end{array}\right] B_{l}^{(k)} \quad(n \geq 1) .
$$

As a counterpart of a generalized poly-Cauchy number, we will define a generalized poly-Bernoulli number $B_{n, a, L}^{(k)}$ by

$$
\frac{\ell^{a-1} \operatorname{Li}_{k}\left(\ell\left(1-e^{-t}\right) ; a-1\right)}{1-e^{-t}}=\sum_{n=0}^{\infty} B_{n, a, L}^{(k)} \frac{t^{n}}{n !},
$$

where $\operatorname{Li}_{k}(z ; a)$ is the generalized polylogarithm function defined by

$$
\operatorname{Li}_{k}(z ; a)=\sum_{m=0}^{\infty} \frac{z^{m}}{(m+a)^{k}},
$$

so that $\operatorname{Li}_{k}(z ; 0)=\operatorname{Li}_{k}(z)$.

Then, $B_{n, a, L}^{(k)}$ can be expressed explicitly in terms of the Stirling numbers of the second kind. Note that $B_{n, 1,(1, \ldots, 1)}^{(k)}=$ $B_{n}^{(k)}$.
Proposition 21. One has

$$
B_{n, a, L}^{(k)}=\sum_{m=0}^{n}\left\{\begin{array}{c}
n \\
m
\end{array}\right\} \frac{(-1)^{n-m} m ! \ell^{m+a}}{(m+a)^{k}} \quad(n \geq 0) .
$$

Proof. By

$$
\frac{\left(e^{t}-1\right)^{m}}{m !}=\sum_{n=m}^{\infty}\left\{\begin{array}{c}
n \\
m
\end{array}\right\} \frac{t^{n}}{n !}
$$

we have

$$
\begin{aligned}
\sum_{n=0}^{\infty} B_{n, a, L}^{(k)} \frac{t^{n}}{n !} & =\frac{\ell^{a}}{\ell\left(1-e^{-t}\right)} \sum_{m=1}^{\infty} \frac{\left(\ell\left(1-e^{-t}\right)\right)^{m}}{(m+a-1)^{k}} \\
& =\ell^{a} \sum_{m=0}^{\infty} \frac{\left(\ell\left(1-e^{-t}\right)\right)^{m}}{(m+a)^{k}} \\
& =\ell^{a} \sum_{m=0}^{\infty} \frac{(-\ell)^{m} m !}{(m+a)^{k}} \sum_{n=m}^{\infty}\left\{\begin{array}{l}
n \\
m
\end{array}\right\} \frac{(-t)^{n}}{n !} \\
& =\sum_{n=0}^{\infty}\left(\sum_{m=0}^{n}\left\{\begin{array}{l}
n \\
m
\end{array}\right\} \frac{(-1)^{n-m} m ! \ell^{m+a}}{(m+a)^{k}}\right) \frac{t^{n}}{n !} .
\end{aligned}
$$

Comparing the coefficients on both sides, we get the result.

For simplicity, we write $c_{n, a, L}^{(k)}=c_{n, a, 1, L}^{(k)}$ and $\widehat{c}_{n, a, L}^{(k)}=\widehat{c}_{n, a, 1, L}^{(k)}$. If $a=\ell=1$, then our results below are reduced to those previous ones.

Theorem 22. For $n \geq 0$, one has

$$
\begin{gathered}
B_{n, a, L}^{(k)}=\sum_{j=1}^{n} \sum_{m=1}^{n} m !\left\{\begin{array}{c}
n \\
m
\end{array}\right\}\left\{\begin{array}{c}
m-1 \\
j-1
\end{array}\right\} c_{j, a, L}^{(k)}, \\
c_{n, a, L}^{(k)}=\sum_{j=1}^{n} \sum_{m=1}^{n} \frac{(-1)^{n-m}}{m !}\left[\begin{array}{c}
n \\
m
\end{array}\right]\left[\begin{array}{c}
m \\
j
\end{array}\right] B_{j, a, L}^{(k)} .
\end{gathered}
$$

Proof. For the first identity,

$$
\begin{aligned}
\text { RHS }= & \sum_{j=1}^{n} \sum_{m=j}^{n} m !\left\{\begin{array}{c}
n \\
m
\end{array}\right\}\left\{\begin{array}{c}
m-1 \\
j-1
\end{array}\right\} \\
& \times \sum_{i=0}^{j}\left[\begin{array}{l}
j \\
i
\end{array}\right] \frac{(-1)^{j-i} \ell^{i+a}}{(i+a)^{k}} \\
= & \sum_{i=1}^{n} \frac{(-1)^{i} \ell^{i+a}}{(i+a)^{k}} \\
& \times \sum_{j=i m=j}^{n} \sum^{n} m !\left\{\begin{array}{c}
n \\
m
\end{array}\right\}\left\{\begin{array}{c}
m-1 \\
j-1
\end{array}\right\}(-1)^{j}\left[\begin{array}{c}
j \\
i
\end{array}\right] \\
= & \sum_{i=1}^{n} \frac{(-1)^{i} \ell^{i+a}}{(i+a)^{k}} \sum_{m=i}^{n} m !\left\{\begin{array}{c}
n \\
m
\end{array}\right\}
\end{aligned}
$$




$$
\begin{aligned}
& \times \sum_{j=i}^{m}(-1)^{j}\left\{\begin{array}{c}
m-1 \\
j-1
\end{array}\right\}\left[\begin{array}{c}
j \\
i
\end{array}\right] \\
= & \sum_{i=1}^{n} \frac{(-1)^{i} e^{i+a}}{(i+a)^{k}} \sum_{m=i}^{n} m !\left\{\begin{array}{c}
n \\
m
\end{array}\right\}(-1)^{m}\left(\begin{array}{c}
m-1 \\
i-1
\end{array}\right) \\
= & \sum_{i=1}^{n} \frac{(-1)^{i} e^{i+a}}{(i+a)^{k}}(-1)^{n} i !\left\{\begin{array}{c}
n \\
i
\end{array}\right\}=\text { LHS. }
\end{aligned}
$$

For the second identity,

$$
\begin{aligned}
& \text { RHS }= \sum_{j=1}^{n} \sum_{m=1}^{n} \frac{(-1)^{n-m}}{m !}\left[\begin{array}{c}
n \\
m
\end{array}\right]\left[\begin{array}{c}
m \\
j
\end{array}\right] \\
& \times \sum_{i=0}^{j}\left\{\begin{array}{l}
j \\
i
\end{array}\right\} \frac{(-1)^{j-i} i ! \ell^{i+a}}{(i+a)^{k}} \\
&= \sum_{m=1}^{n} \frac{(-1)^{n-m}}{m !}\left[\begin{array}{c}
n \\
m
\end{array}\right] \\
& \times \sum_{j=0}^{n}\left[\begin{array}{c}
m \\
j
\end{array}\right] \sum_{i=0}^{j}\left\{\begin{array}{l}
j \\
i
\end{array}\right\} \frac{(-1)^{j-i} i ! \ell^{i+a}}{(i+a)^{k}} \\
&= \sum_{m=1}^{n} \frac{(-1)^{n-m}}{m !}\left[\begin{array}{c}
n \\
m
\end{array}\right] \\
& \times \sum_{i=0}^{n} \frac{(-1)^{i} i ! \ell^{i+a}}{(i+a)^{k}} \sum_{j=i}^{n}(-1)^{j}\left[\begin{array}{c}
m \\
j
\end{array}\right]\left\{\begin{array}{l}
j \\
i
\end{array}\right\} \\
&= \sum_{m=0}^{n}\left[\begin{array}{l}
n \\
m
\end{array}\right] \frac{(-1)^{n-m} \ell^{m+a}}{(m+a)^{k}}=\text { LHS. } \\
&= \sum_{m=0}^{n} \frac{(-1)^{n-m}}{m !}\left[\begin{array}{l}
n \\
m
\end{array}\right] \\
& \times \frac{(-1)^{m} m ! \ell^{m+a}}{(m+a)^{k}}(-1)^{m} \\
& \\
&
\end{aligned}
$$

Note that $\left[\begin{array}{c}m \\ 0\end{array}\right]=0(m \geq 1)$ and $\left[\begin{array}{c}m \\ j\end{array}\right]=0(j>m)$, and

$$
\sum_{j=i}^{m}(-1)^{m-j}\left[\begin{array}{c}
m \\
j
\end{array}\right]\left\{\begin{array}{l}
j \\
i
\end{array}\right\}= \begin{cases}1 & (i=m) \\
0 & (i \neq m)\end{cases}
$$

Similarly, concerning

$$
\widehat{c}_{n, a, L}^{(k)}=(-1)^{n} \sum_{m=0}^{n}\left[\begin{array}{c}
n \\
m
\end{array}\right] \frac{e^{m+a}}{(m+a)^{k}} \quad(n \geq 0)
$$

as a generalization of poly-Cauchy numbers of the second kind $\widehat{c}_{n}^{(k)}$, we have the following.
Theorem 23. One has

$$
\begin{aligned}
& B_{n, a, L}^{(k)}=(-1)^{n} \sum_{j=1}^{n} \sum_{m=1}^{n} m !\left\{\begin{array}{c}
n \\
m
\end{array}\right\}\left\{\begin{array}{c}
m \\
j
\end{array}\right\} \widehat{c}_{j, a, L}^{(k)}, \\
& \widehat{c}_{n, a, L}^{(k)}=(-1)^{n} \sum_{j=1}^{n} \sum_{m=1}^{n} \frac{1}{m !}\left[\begin{array}{c}
n \\
m
\end{array}\right]\left[\begin{array}{c}
m \\
j
\end{array}\right] B_{j, a, L}^{(k)} .
\end{aligned}
$$

Remark 24. If $a=\ell=1$, these results are reduced to the identities in Theorems 3.2 and 3.1 in [9], respectively.

\section{Acknowledgments}

This work was partly done when the first author visited Eszterházy Károly College in September 2012 and Kasetsart University in October 2012. He thanks both institute and the college for their hospitality. This work was completed when the third author visited Hirosaki University in November 2012. This work was supported in part by the Grant-in-Aid for Scientific research (C) (no. 22540005), the Japan Society for the Promotion of Science.

\section{References}

[1] T. Komatsu, "Poly-Cauchy numbers," Kyushu Journal of Mathematics, vol. 67, no. 1, pp. 143-153, 2013.

[2] L. Comtet, Advanced Combinatorics, D. Reidel Publishing Co., Dordrecht, The Netherlands, 1974.

[3] D. Merlini, R. Sprugnoli, and M. C. Verri, "The Cauchy numbers," Discrete Mathematics, vol. 306, no. 16, pp. 1906-1920, 2006.

[4] R. L. Graham, D. E. Knuth, and O. Patashnik, Concrete Mathematics, Addison-Wesley, Reading, Mass, USA, 2nd edition, 1994.

[5] “The On-Line Encyclopedia of Integer Sequences," http://oeis .org

[6] M. Kaneko, "Poly-Bernoulli numbers," Journal de Théorie des Nombres de Bordeaux, vol. 9, no. 1, pp. 221-228, 1997.

[7] T. Komatsu, "Poly-Cauchy numbers with a $q$ parameter," Ramanujan Journal, vol. 31, no. 3, pp. 353-371, 2013.

[8] T. Komatsu and L. Szalay, "Shifted poly-Cauchy numbers," (preprint).

[9] T. Komatsu and F. Luca, "Some relationships between polyCauchy numbers and poly-Bernoulli numbers," Annales Mathematicae et Informaticae, vol. 41, pp. 99-105, 2013. 


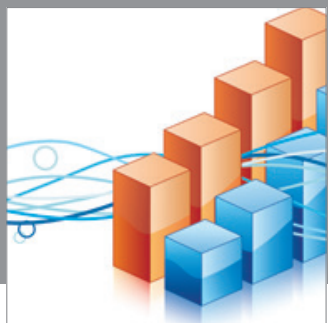

Advances in

Operations Research

mansans

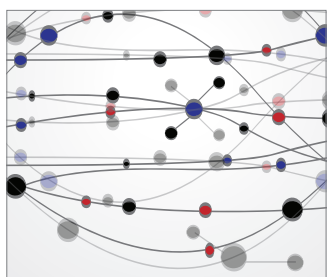

The Scientific World Journal
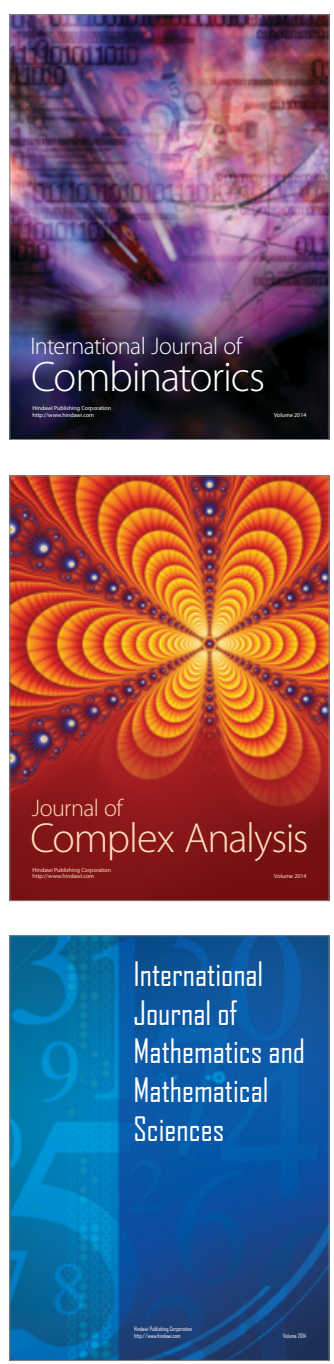
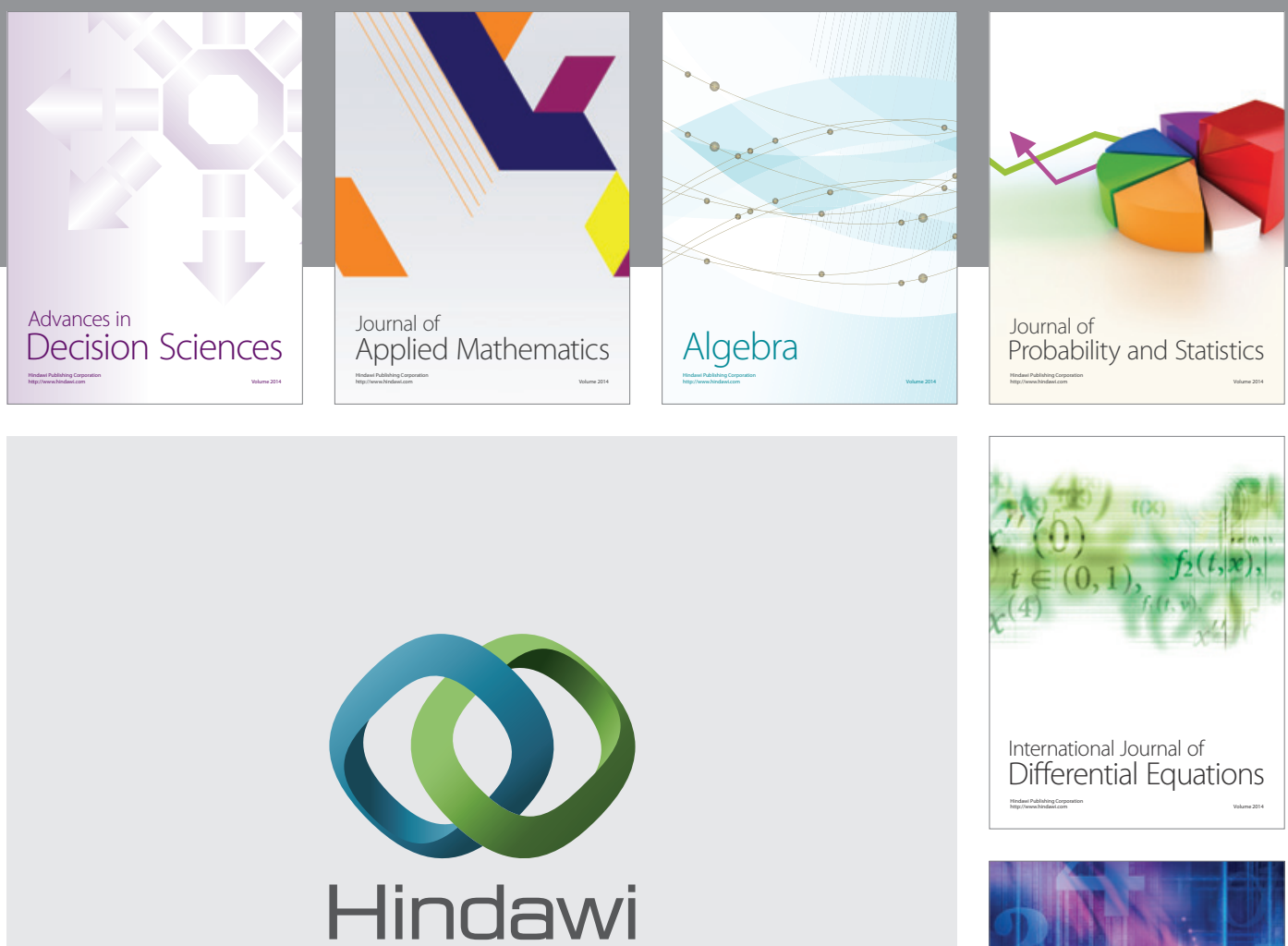

Submit your manuscripts at http://www.hindawi.com
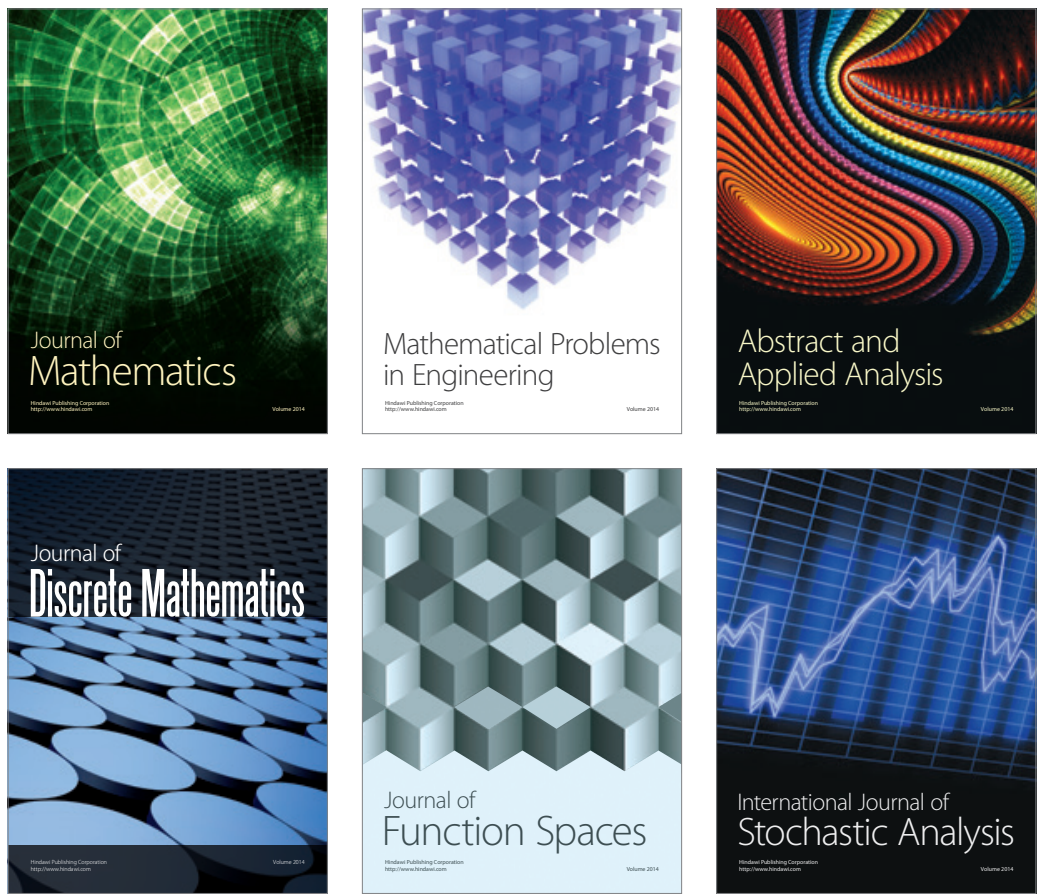

Journal of

Function Spaces

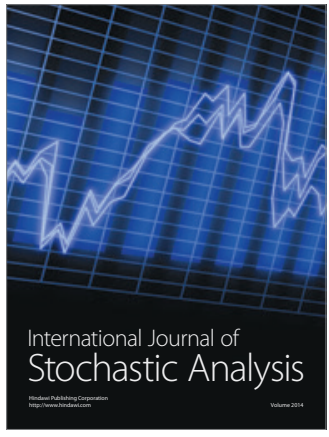

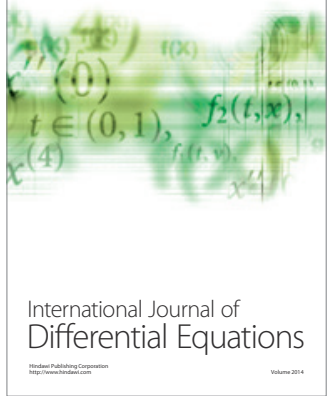
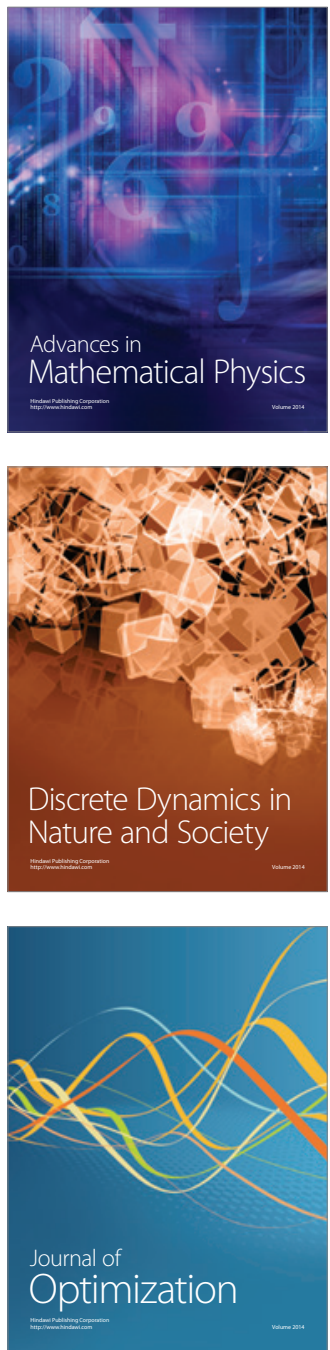